\title{
2 | Conséquences environnementales
}

Deux aspects sont à considérer : ceux qui concernent directement les conséquences des rejets radioactifs sur la faune et la flore et ceux qui résultent de la contamination des productions locales. Ce dernier aspect est important car l'impact des rejets radioactifs affecte gravement la valeur économique des territoires affectés, nuisant à la commercialisation des produits quand bien même les normes qui en limitent les dangers sont respectées.

\subsection{Impacts de l'exposition aux radiations sur la faune et la flore terrestres}

\subsubsection{Aspects généraux}

Bien qu'il soit impossible de déterminer les effets des radiations pour toutes les espèces connues, la figure ci-dessous établie par l'UNSCEAR permet de se rendre compte que la sensibilité aux radiations ionisantes est différente entre les animaux, les végétaux et les micro-organismes:

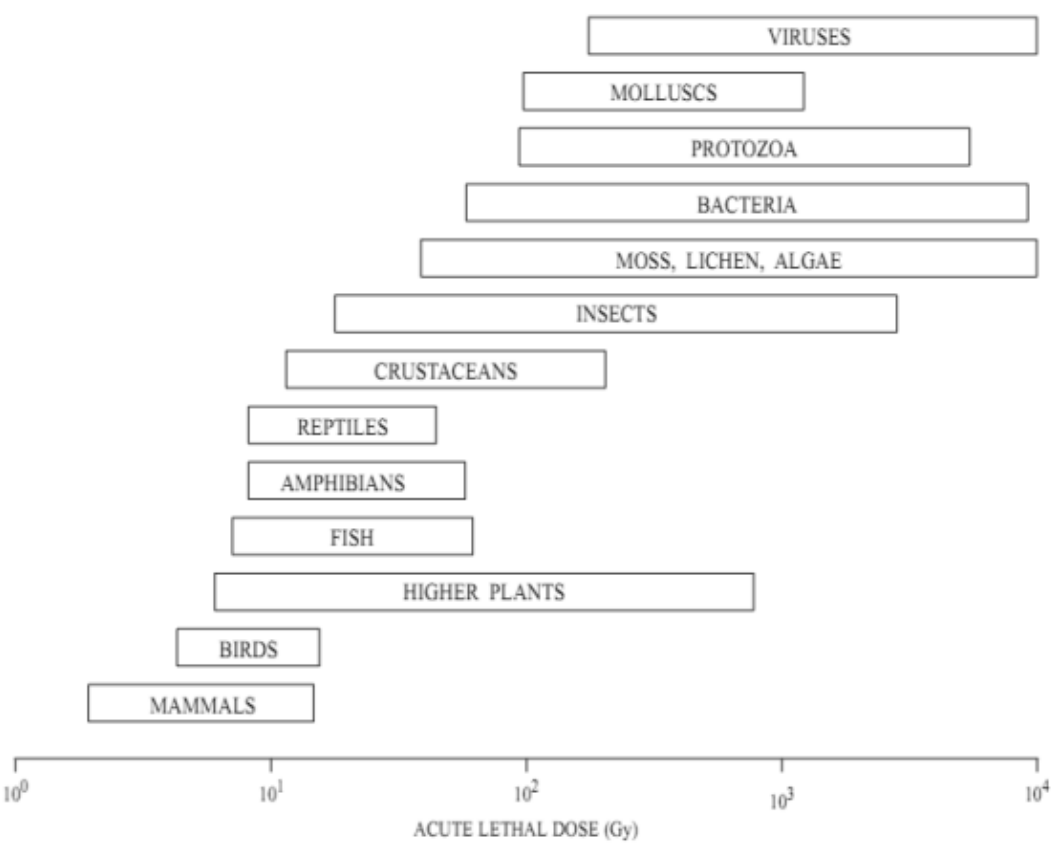


Ces données établies par I'UNSCEAR donnent sens à la position proférée par la CIPR dans ses anciennes recommandations; «qui protège l'homme protège l'environnement ». Malgré certaines contestations, la démonstration inverse $n^{\prime}$ est toujours pas faite.

Pour les plantes, par exemple, il est possible de déterminer les effets sur différentes fonctions biologiques exprimées en fonction de la dose létale, en examinant la figure suivante.

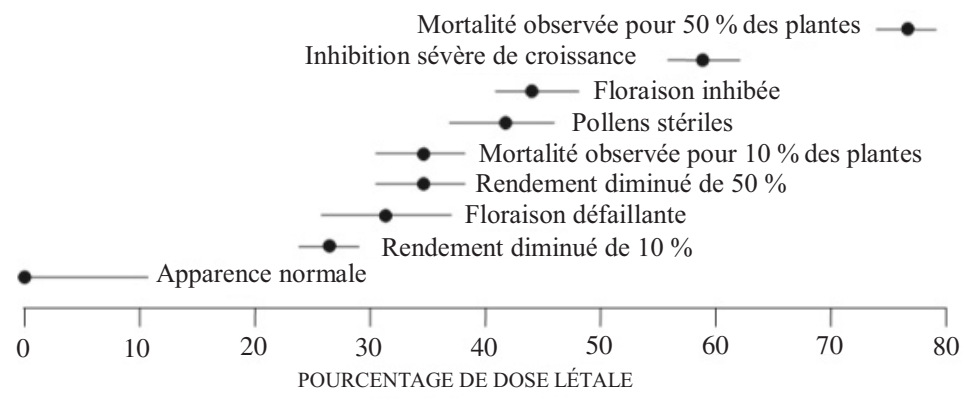

À partir des conséquences de l'accident de Tchernobyl, il est possible $d^{\prime}$ avoir une description des impacts des radiations sur la flore et la faune de l'écosystème entourant la centrale nucléaire de Tchernobyl.

\subsubsection{Impact à̀ Tchernobyl}

- Les doses élevées de rayonnements reçues pendant quelques semaines après l'accident, observées pour des dépôts de $0,7-3,9 \mathrm{GBq} \mathrm{m}^{-2}$, ont conduit à des effets délétères dans une zone de quelques dizaines de kilomètres de diamètre (zone évacuée de $30 \mathrm{~km}$ autour de la centrale de Tchernobyl).

- Un accroissement de la mortalité a été constaté dans cette zone sur les animaux, les invertébrés du sol et sur les conifères qui sont les végétaux les plus sensibles (d'où l'expression « forêt rousse » utilisée pour cette zone par les médias). Un affaiblissement reproductif a été constaté aussi bien chez les plantes que les animaux sauvages (mammifères, oiseaux, etc.).

- Après une atteinte à l'équilibre écologique de la plupart des espèces, en quelques années, dans la zone évacuée de $30 \mathrm{~km}$ autour de la centrale, les champs abandonnés et les forêts sont devenus des refuges pour de nombreuses espèces de plantes ou d'animaux sauvages qui ont pu occuper les niches écologiques abandonnées et s'y reproduire. 
- Pour les invertébrés du sol, dans la zone des $30 \mathrm{~km}$, après une réduction drastique par un facteur 30 de leurs populations, les populations ont retrouvé environ dix ans après leur diversité initiale.

De l'ensemble des observations effectuées depuis plus de vingt ans peuvent être tirés les enseignements suivants :

- pour les plantes terrestres les plus sensibles (conifères), des doses chroniques de l'ordre de 400 microGy/h (10 mGy/i) ont des effets très faibles, alors qu'elles sont sans effets sur les autres types de végétaux ;

- pour la plupart des communautés aquatiques (eaux douces), les doses chroniques inférieures à $400 \mathrm{microGy} / \mathrm{h}(10 \mathrm{mG} / \mathrm{i})$, pour les individus les plus exposés, ne semblent pas susceptibles de produire des effets délétères ;

- pour les animaux terrestres les plus sensibles (mammifères), des doses chroniques à 40 microGy/h (1 mGy/i) ne semblent pas avoir d'impact sur leurs capacités reproductives. Il en est de même pour les oiseaux, les reptiles et les invertébrés qui sont en général moins radiosensibles que les mammifères.

Les mutations génétiques ou somatiques qui peuvent être produites par des niveaux bas d'exposition peuvent n'avoir aucun impact ou un niveau $d^{\prime}$ 'impact faible au niveau des biocénoses (équilibres des populations animales et végétales), par suite de la sélection naturelle ou du fait d'échanges avec les populations présentes dans les milieux voisins non contaminés.

\subsubsection{Situation à Fukushima}

Bien que globalement la radioactivité rejetée à Fukushima soit $10 \%$ de celle de Tchernobyl, les niveaux de radioactivité au sol dans les zones les plus contaminées sont comparables. Les mêmes impacts peuvent donc en être attendus. Cependant, les deux accidents ne sont pas de même nature. À Fukushima, seule la dispersion d'éléments volatils a eu lieu suite à la fusion du cœur du réacteur; il n'y a pas eu de dispersion de fragments de combustible nucléaire. On peut s'attendre cependant à ce que la solubilité des radionucléides contaminants soit supérieure à celle des mêmes radionucléides piégés dans des particules de combustible.

\subsection{Impact sur les produits végétaux de consommation : les différents modes de déposition}

\subsubsection{Le transfertt aux végétaux}

Après l'accident, les radionucléides se déposent par gravité (voie sèche) ou par entraînement par l'eau de pluie ou la neige (voie humide). Cette deuxième 


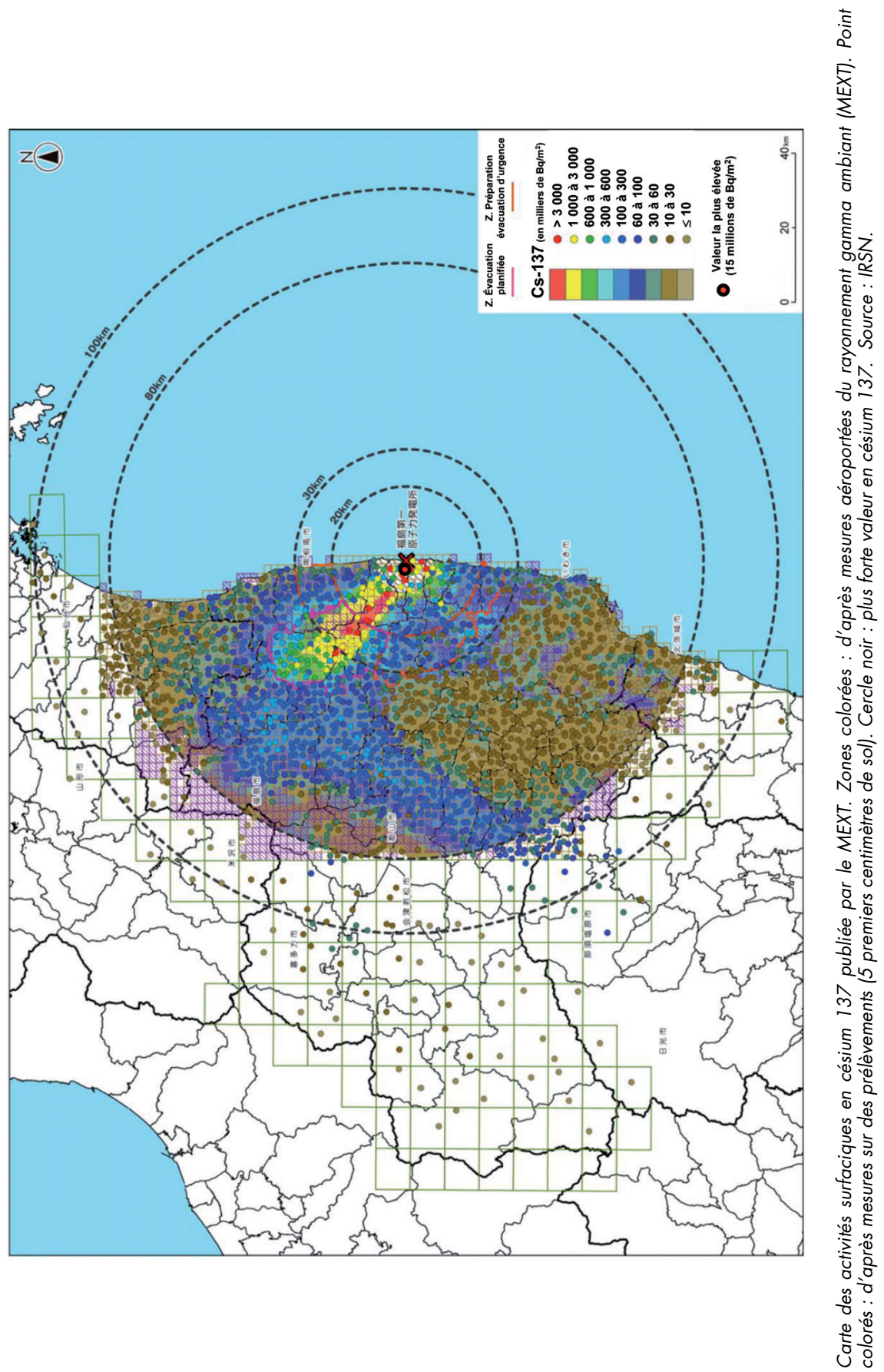


voie est beaucoup plus importante que la première et conduit à des dépositions très différentes selon la pluviométrie. Cela s'est caractérisé par une contamination « en taches de léopard ».

Le transfert des radionucléides déposés sur la végétation est essentiellement foliaire. II a lieu l'année de la contamination. La surface foliaire est un élément déterminant. Dans un deuxième temps, après migration lente dans le sol, le transfert devient racinaire. Le transfert foliaire est plus élevé que le transfert racinaire, c'est pourquoi la contamination des plantes est maximale l'année de l'accident. Le transfert foliaire dépend essentiellement du stade de développement de la plante. La vitesse de migration dépend essentiellement de la nature des sols. Elle est très lente dans les prairies et sous-bois; la contamination des produits de la forêt, champignons et baies, restera donc importante durant de nombreuses années.

Suite aux retombées de l'accident de Fukushima, parmi les productions agricoles, les radioactivités les plus élevées n'ont concerné que les légumes, principalement les légumes à feuilles (épinards notamment). La filière laitière a été touchée modérément et la contamination de la viande, à cinétique plus lente, concerne principalement des animaux alimentés avec des fourrages stockés en extérieur dans des zones où les dépôts ont été très importants.

Trois types de produits ont présenté une contamination significative : les pousses de bambou, les feuilles de thé crues ou raffinées et les abricots du Japon. Ci-dessous figure un extrait de la synthèse faite par l'IRSN le 13 juillet 2011 , concernant la contamination de la chaîne alimentaire au Japon.

"Les activités massiques mesurées $\left({ }^{134} \mathrm{Cs}+{ }^{137} \mathrm{Cs}\right)$ peuvent dépasser de l'ordre de $1000 \mathrm{~Bq} / \mathrm{kg}$ frais pour le thé issu de la première récolte (1 $330 \mathrm{~Bq} / \mathrm{kg}$ frais à Kanagawa et $981 \mathrm{~Bq} / \mathrm{kg}$ frais à Shizuoka le 21 juin) et de $2000 \mathrm{~Bq} / \mathrm{kg}$ frais pour les pousses de bambou $(2060 \mathrm{~Bq} / \mathrm{kg}$ à Minamisoma et $1070 \mathrm{~Bq} / \mathrm{kg}$ à Souma le 23 juin). Ces niveaux de contamination pourraient correspondre à des dépôts de césium relativement modérés, estimés à quelques dizaines de milliers de $\mathrm{Bq} / \mathrm{m}^{2}$, qui ont pu se former jusqu'à plusieurs centaines de kilomètres de la centrale de Fukushima Dai-ichi. Il est donc possible que des contaminations beaucoup plus importantes de ces produits puissent être observées dans des productions en provenance de zones plus touchées par les retombées radioactives. Les mesures effectuées sur les feuilles de thé et les pousses de bambou sont relatives aux premières récoltes après les dépôts... les feuilles de thé qui ont fait l'objet d'une seconde coupe sont moins contaminées que celles de la première coupe : les activités en césium mesurées en juin varient entre 29 et $306 \mathrm{~Bq} / \mathrm{kg}$ frais. 
Toutefois, la consommation de ces denrées n'est pas immédiate et les produits conditionnés issus des premières récoltes sont susceptibles de perdurer sur le marché durant des mois.

Les abricots du Japon (umé) sont les fruits d'arbres à floraison très précoce, qui étaient probablement déjà en fleurs mi-mars... Les résultats de mesure les plus récents dans les abricots japonais récoltés dans la préfecture de Fukushima varient entre 137 et $700 \mathrm{~Bq} / \mathrm{kg}$ frais pour les isotopes 134 et 137 du césium... Cette contamination résulte de l'interception des dépôts radioactifs par les organes végétatifs présents à ce moment-là. II est probable que la contamination des abricots japonais ne se limitera pas à cette première récolte. Toutefois ces productions devraient être, dans l'avenir, moins sensibles à cette pollution radioactive que les feuilles de thés ou les pousses de bambous. »

Les conséquences immédiates de l'accident de Fukushima sur la chaîne alimentaire au Japon doivent donc être analysées en considérant l'influence de la date d'occurrence des dépôts qui les a considérablement limitées, compte tenu de la saison. Les conséquences à long terme doivent en revanche tenir compte du niveau de contamination des sols et des coefficients de transferts des contaminants (principalement le césium) vers les parties consommées des végétaux.

\subsubsection{Contamination de la faune aquatique}

L'IRSN a publié le 11 juillet 2011 (et réactualisé le 26 octobre 2011 ) une synthèse de la contamination en milieu marin. Une forte contamination radioactive du milieu marin s'est produite après l'accident de Fukushima. Elle a pour origine le déversement des eaux contaminées qui ont servi à refroidir les réacteurs endommagés et dans une moindre mesure les retombées dans l'océan d'une partie des radionucléides rejetés dans l'atmosphère les dix premiers jours qui ont suivi l'accident, lorsque les vents étaient dirigés vers la mer.

Pour l'iode 131, qui se fixe dans la faune et la flore marine, la concentration a rapidement décru en raison de sa période radioactive courte, à partir de valeurs maximales atteignant plusieurs centaines de milliers de Bq/L à proximité du canal de décharge des eaux de refroidissement.

La concentration pour les deux isotopes du césium a atteint plusieurs dizaines de milliers de $\mathrm{Bq} / \mathrm{L}$ à proximité de la côte pour décroître rapidement fin avril aux environs de $100 \mathrm{~Bq} / \mathrm{L}$. 
Les isotopes radioactifs solubles du césium ont été transportés par les courants marins et se sont dispersés dans les masses d'eaux océaniques. La diminution de la concentration du césium dans le milieu marin proche de la centrale se fait avec une période de onze jours.

En août 2011, l'AIEA a publié une synthèse des conséquences sur les produits de la pêche côtière. Seuls les lançons ont fait l'objet de dépassements réguliers des valeurs limites, atteignant plus de $10000 \mathrm{~Bq} / \mathrm{kg}$ d'iode 131 dans une zone de pêche située à $40-60 \mathrm{~km}$ au sud de Fukushima. Toutes les pêcheries ont été gelées dans la préfecture de Fukushima et, partiellement, au sud pour les pêcheries d'lbaraki.

\subsection{Décontamination des milieux}

\subsubsection{Réduction de la contamination en milieu bâti}

L'objectif principal des actions de réduction d'une contamination faisant suite à un accident nucléaire est $\mathrm{d}^{\prime}$ 'améliorer la situation radiologique de l'environnement et de réduire en conséquence l'exposition des populations qui seraient maintenues sur place ou qui se réinstalleraient après un éloignement temporaire. En aucun cas ces actions ne permettront un retour à l'état initial, c'est-à-dire une décontamination totale. La prise en compte de cette dimension impose que la population fasse confiance aux dispositions prises par les pouvoirs publics.

\subsubsection{Voies d'exposition majeures en milieu bâti et actions de réduction de la confamination}

C'est la contamination des toits des habitations, des routes, des trottoirs et de la végétation «urbaine » qui contribue principalement à l'irradiation externe des populations, l'importance respective de chacune de ces sources d'exposition variant évidemment selon le mode de vie (habitat individuel ou collectif) et le type de dépôt (humide ou sec). Les phénomènes de concentration dus au recueil par l'eau de pluie des particules déposées (gouttières pour le toit des habitations, égouts pour les grandes surfaces urbaines) sont à prendre en compte. L'accident de Tchernobyl a montré qu'il fallait être vigilant également avec les systèmes de ventilation des habitations et immeubles.

Les actions possibles pour la réduction de la contamination présentent des contraintes spécifiques de mise en œuvre qui dépendent de la disponibilité opérationnelle des moyens techniques et du temps écoulé depuis le début de 
I'accident. Ces actions, qui peuvent apporter une réduction d'un facteur 2 à 3, sont détaillées dans l'annexe 14 « Réduction de la contamination en milieu bâti ».

\subsubsection{Mesures de restauration des sols contaminés}

L'expérience acquise à Tchernobyl a montré qu'on ne pouvait restaurer complètement la qualité des sols: l'essentiel est de redonner aux territoires contaminés une valeur économique. Ce programme très complexe nécessite plusieurs étapes, détaillées dans l'annexe 15. En résumé, les actions supposent une bonne connaissance des terrains contaminés, la mise au point de techniques mécaniques et physicochimiques de décontamination, le choix de cultures appropriées soit pour leurs propriétés couvrantes, soit pour leur aptitude à concentrer ou ignorer le césium, soit pour leur usage industriel retardé, ou en tant que biomasse pour la production d'énergie dans des installations dédiées.

\subsection{Déchets contaminés}

Le traitement des déchets est un problème politique majeur au Japon, il concerne d'une part les débris du tsunami et les liquides récupérés et stockés "temporairement» sur un site qui sera créé à proximité de la centrale et $d^{\prime}$ 'autre part tous les résidus résultant du traitement des surfaces dans les zones à réhabiliter.

Les Japonais se sont fixés comme objectif de traiter les zones où l'activité peut conduire à des doses de $5 \mathrm{mSv} / \mathrm{an}$ (correspondant à $1 \mu \mathrm{Sv}$ par heure). Pour les zones où l'exposition est inférieure à $5 \mathrm{mSv}$, le gouvernement japonais estime que cette activité évoluera rapidement à un niveau inférieur à la dose limite pour les populations $(1 \mathrm{mSv} / \mathrm{an}$ avec le temps et les conditions météorologiques, pluie et vent).

Pour les zones évacuées, toujours dans l'optique de récupération de territoires, des décapages importants sur une profondeur de $5 \mathrm{~cm}$ où se situent les césiums seront nécessaires; ils aboutiront à des volumes de déchets de l'ordre de 400 tonnes à l'hectare pour une division par 4 à 5 du facteur de dose. Ce travail de décontamination par décapage n'est pas envisagé à ce jour pour les forêts (70\% des zones à traiter). Le seul ramassage des feuilles et un élagage pourraient suffire. De plus, le volume de déchets contaminés pourrait être réduit par incinération, avec cependant une concentration de la contamination. 
Dans les zones qui n'ont pas été évacuées, il sera procédé à un diagnostic précis de l'état des surfaces, et les supports les plus contaminés seront enlevés et stockés selon un concept proche de notre gestion des déchets de très faible activité. II sera procédé à des labours plus ou moins profonds, à l'utilisation d'engrais, etc., tous procédés testés à Tchernobyl et résumés dans le rapport UNSCEAR de $2011^{15}$.

Le ministère de l'Environnement japonais évalue la surface à traiter à $2400 \mathrm{~km}^{2}$, répartis autour de Fukushima, et le volume de terres contaminées à entreposer à 29 millions de $\mathrm{m}^{3}$.

Pour mémoire, à Tchernobyl, dans les zones les plus contaminées autour de la centrale accidentée, les déchets avaient été évacués et enfouis dans la zone de la «forêt rousse ». Cette opération avait été réalisée dans l'optique d'une amélioration de l'accessibilité à la centrale, non pour rendre viable cette zone contaminée.

15 UNSCEAR Report 2008: Sources and effects of ionizing radiation, Vol II, Annex E: Effects of ionizing radiation on non human biota, United Nations, New-York 2011. 